\title{
Usefulness of Patient-Reported Outcome Measures and Anorectal Physiologic Tests in Predicting Clinical Outcome for Fecal Incontinence
}

\author{
Chang-Nam Kim \\ Department of Surgery, Eulji University Hospital, Eulji University School of Medicine, Daejeon, Korea
}

\section{See Articles on Page 319-326}

Fecal incontinence (FI) is one of the most intractable diseases for colorectal surgeons. Although many reports have been published, the pathophysiology of FI is not yet fully understood, and its cause is often difficult to identify. Treatment of FI includes conservative and surgical treatments with the aim of improving symptoms and patients' quality of life (QoL). Surgical treatment for FI is generally considered in a small number of patients who have failed to respond to conservative treatment. However, the choice of conservative or surgical treatment has depended on the decision of the individual physician, as few studies have revealed objective tools for the selection of surgical treatment.

Numerous surgical methods, including anal sphincter repair, an artificial anal sphincter, and sacral nerve stimulation (SNS), have been proposed to treat FI. Wald [1] reported on the uncertain effect of the traditional surgical technique, anal sphincter repair, and noted that short-term results showed improvement in up to $85 \%$ of patients with FI while long-term results showed a failure rate of more than $50 \%$ after 40 to 60 months, which increased further thereafter $[2,3]$. The author also reported that the effects of treatment deteriorated over time, especially in older patients. O'Brien et al. [4], in a prospective, randomized, controlled study, reported the effect of an artificial anal sphincter on patients with severe FI. In the artificial anal sphincter group (7 patients) and the control group (7 patients), the Wexner Incontinence Scores were com-

Correspondence to: Chang-Nam Kim, M.D.

Department of Surgery, Eulji University Hospital, Eulji University School of Medicine, 95 Dunsanseo-ro, Seo-gu, Daejeon 35233, Korea

Tel: +82-42-259-1335, Fax: +82-42-259-1335

E-mail: kimcn7@gmail.com

ORCID code: https://orcid.org/0000-0001-7781-9119

(C) 2019 The Korean Society of Coloproctology

This is an open-access article distributed under the terms of the Creative Commons Attribution NonCommercial License (http://creativecommons.org/licenses/by-nc/4.0) which permits unrestricted noncommercial use, distribution, and reproduction in any medium, provided the original work is properly cited. pared before and after 6 months of treatment. At 6 months, a significant difference was found between the groups (artificial anal sphincter group vs. control group: from 19.0 to 4.8 vs. from 17.1 to $14.3, \mathrm{P}=0.002)$. However, this was the result of only 14 patients. Benezech et al. [5] mentioned that the artificial sphincter had a high rate of complications, but a moderate effect, which, in turn, limited its use for treating patients with FI. Thaha et al. [6] reported a systemic review on the efficacy of SNS for treating patients with FI. The authors selected all randomized trials assessing the effects of SNS for treating FI in adults. They found limited evidences from the trials and suggested that a proportion of patients with FI could be improved by using SNS. Therefore, the authors emphasized the need for strict high-quality randomized trials to better assessment the effects of SNS.

At present, the evaluation of FI also requires high-quality evidence. Although studies have predicted the effectiveness of each treatment of FI, the tools used to assess the effectiveness were not integrated and varied from subjective patient-reported outcome measures (PROMs) to physiologic tests, including anorectal manometry (ARM). Whether these subjective scoring systems or physiologic tests including ARM would be better as a measure to help predict the appropriate treatment for FI is not clear yet. What is the usefulness of PROMs and anorectal physiologic tests in predicting the clinical outcome for patients with FI?

Ramage et al. [7] conducted a very meaningful study, perhaps the first study using ARM to predict the need for surgery in patients with FI. The authors assessed the usefulness of ARM and PROMs in predicting the need for surgery in 276 patients with FI. Two hundred twenty-eight patients $(82.6 \%)$ underwent conservative treatment, and 48 patients (17.4\%) underwent surgery. On binomial regression analyses, age, male sex, ARM including maximal resting pressure, 5-second squeeze increment, threshold volume to distension, urge volume to distension, all domains of the Birmingham Bowel and Urinary Symptoms Questionnaire (BBUSQ), the Wexner Incontinence Score, and all domains of the Short Form 36 (SF-36) were factors predictive of the need for surgery. Receiver operating characteristic curve analyses showed that 
age, 5-second squeeze endurance in ARM only, PROMs including the incontinence of the BBUSQ domains, and five domains (physical function, role physical, mental health, social function, general health) of SF-36 were meaningful discriminators of the need for surgery. The authors concluded that PROMs and only 5-second squeeze endurance in ARM were reliable factors predictive of the need for surgery in patients with FI.

Deutekom et al. [8] compared changes in various outcome measures (descriptive, severity like FI scoring systems, impact like QoL questionnaires, and diagnostic measures) before and after physiotherapy to patients' subjective perceptions of relief. In the correlations between the changes in various outcome measures, the correlation between the changes in the Vaizey and the Wexner scores was high $(\mathrm{P}<0.01)$. The correlation between the changes in the severity scores and the changes in the maximum incremental squeeze pressure was moderate $(\mathrm{P}<0.05)$. In the relation between various outcome measures and patients' perception (worse or equal, better, and much better), no significant differences in the changes in the resting pressure, maximal incremental squeeze pressure, and utility values were found among the three groups. However, significant differences were found in the Vaizey and the Wexner scores $(\mathrm{P}<0.001)$, in the impact measures including FI $(\mathrm{P}$ $=0.011)$ and disease-specific psychosocial functioning $(\mathrm{P}=$ 0.022). Post hoc tests revealed that in subjective perception, the changes in severity measures (Vaizey and Wexner) were more correlated than the changes in FI and disease-specific psychosocial functioning. Therefore, the authors concluded that severity outcome measures, including the Vaizey and the Wexner scores, had the best correlations to patients' relief.

Quezada et al. [9] reported the roles of preoperative anal physiology testing (ARM and pudendal nerve terminal motor latency [PNTML]) and transanal ultrasonography (TAUS) in predicting clinical outcome when using SNS to treat patients with FI. Sixty patients underwent SNS. Three months after the SNS treatment, no correlations were found between the Wexner Incontinence Scores and the test results of ARM, PNTML, and TAUS. Therefore, the authors concluded that ARM, PNTML, and TAUS could not predict clinical outcome when using SNS to treat patients with FI.

In conclusion, the aforementioned studies reported that PROMs showed useful results predicting the clinical outcome for patients with FI while anorectal physiologic tests showed inconsistent results. FI is a physically and mentally devastating disease and places a huge burden on patients, their families, their caregivers, and society [10]. Therefore, every effort should be made, through high- quality randomized trials, to establish clear guidelines for assessing and diagnosing FI and for establishing both treatments for FI and tools for predicting the clinical outcomes when using those treatments.

\section{CONFLICT OF INTEREST}

No potential conflicts of interest relevant to this article were reported.

\section{REFERENCES}

1. Wald A. Update on the management of fecal Incontinence for the gastroenterologist. Gastroenterol Hepatol 2016;12:155-64.

2. Gutierrez AB, Madoff RD, Lowry AC, Parker SC, Buie WD, Baxter NN. Long-term results of anterior sphincteroplasty. Dis Colon Rectum 2004;47:727-31.

3. Halverson AL, Hull TL. Long-term outcome of overlapping anal sphincter repair. Dis Colon Rectum 2002;45:345-8.

4. O'Brien PE, Dixon JB, Skinner S, Laurie C, Khera A, Fonda D. A prospective, randomized, controlled clinical trial of placement of the artificial bowel sphincter (Acticon Neosphincter) for the control of fecal incontinence. Dis Colon Rectum 2004;47:1852-60.

5. Benezech A, Bouvier M, Vitton V. Faecal incontinence: current knowledges and perspectives. World J Gastrointest Pathophysiol 2016;15:59-71.

6. Thaha MA, Abukar AA, Thin NN, Ramsanahie A, Knowles CH. Sacral nerve stimulation for faecal incontinence and constipation in adults. Cochrane Database Syst. Rev 2015;8:CD004464.

7. Ramage L, Qiu S, Yeap Z, Simillis C, Kontovounisios C, Tekkis P, et al. Anorectal manometry versus patient-reported outcome measures as a predictor of maximal treatment for fecal incontinence. Ann Coloproctol 2019;35:319-26.

8. Deutekom M, Terra MP, Dobben AC, Dijkgraaf MGW, Felt-Bersma RJF, Stoker J, et al. Selecting an outcome measure for evaluating treatment in fecal incontinence. Dis Colon Rectum 2005;48: 2294-301.

9. Quezada Y, Whiteside JL, Rice T, Karram M, Rafferty JF, Paquette IM. Does preoperative anal physiology testing or ultrasonography predict clinical outcome with sacral neuromodulation for fecal incontinence? Int Urogynecol J 2015;26:1613-7.

10. Meyer I, Richter HE. Impact of fecal incontinence and its treatment on quality of life in women. Womens Health 2015;11:225-38. 